\title{
MONTAÑAS, LINTERNAS, TELESCOPIOS Y RELOJES: GALILEO Y EL PROBLEMA DE LA PROPAGACIÓN SUCESIVA DE LA LUZ ${ }^{1}$
}

\author{
MOUNTAINS, LANTERNS, TELESCOPES, \\ AND CLOCKS: GALILEO AND THE PROBLEM \\ OF SUCCESSIVE PROPAGATION OF LIGHT
}

Carlos González-Sierra²

$\square$ s importante destacar que cuando en la historia de la física se hace referencia al problema de si la luz se transmite de manera instantánea o si requiere de tiempo ${ }^{3}$-que se abreviará aquí como el problema de la propagación sucesiva de la luz- siempre destacan ciertos personajes: por un lado, Armand Hippolyte Louis Fizeau y Jean Bernard León Foucault, quienes en el siglo XIX lograron medir por primera vez de forma precisa la velocidad de la luz; y por otro, los astrónomos Ole Christensen Roemer y James Bradley, quienes en los siglos XVII y XVIII -respectivamente- fueron los primeros en dar una respuesta derivada

1 Esta conferencia se deriva de la tesis Midiendo el movimiento de los cuerpos celestes, conociendo la luz: aspectos epistémicos y cognitivos de la medición científica, presentada en el año 2015 en el marco de los estudios de la Maestría en Filosofía de la Ciencia en la Universidad Nacional Autónoma de México.

2 Magíster en Filosofía de la Ciencia (Historia de la Ciencia) de la Universidad Nacional Autónoma de México (2015). Profesional en Filosofía y Letras de la Universidad Pontificia Bolivariana (2012). Docente de cátedra de la Escuela de Teología, Filosofía y Humanidades de la Universidad Pontificia Bolivariana.

Correo electrónico: carlosandres.gonzalez@alfa.upb.edu.co. orcid.org/0000-0001-8828-6004

3 Tradicionalmente, el problema de si la luz se transmite instantáneamente o si requiere de tiempo es presentado como equivalente al de si la luz tiene una velocidad finita o infinita. Sin embargo, como se verá a lo largo de la conferencia ambos problemas tienen una diferencia conceptual: es posible enfrentar el primero sin el concepto de velocidad de la luz -como es el caso de Galileo (cf. Cassini)-, mientras que el segundo implica dicha noción, siendo el centro de la investigación la determinación de un valor numérico. 
de trabajos observacionales de que la luz sí requiere de tiempo en su transmisión, esto es, que su propagación es sucesiva. Entre estos nombres, aparece siempre de forma algo etérea Galileo Galilei como el primero que propuso un enfoque experimental para dar respuesta a esta pregunta.

Se afirma que la figura del filósofo natural italiano resulta un tanto etérea en la historia del problema de la propagación sucesiva de la luz por dos razones: la primera, porque en palabras del mismo Galileo, el resultado de su experimento fue no-concluyente, esto es, la realización del experimento no le permitió llegar a una conclusión robusta sobre ninguna de las dos opciones; y la segunda, porque su experimento -considerado desde una perspectiva anacrónica- ha sido etiquetado como "inocente", debido a que era imposible saber de antemano la magnitud de la velocidad de la luz. En otras palabras, aunque el experimento metodológicamente es adecuado, su realización en tiempos de Galileo resultaba casi imposible debido a los requerimientos técnicos que implica el enfrentarse a semejante velocidad.

A pesar de lo anterior, la figura de Galileo siempre aparece desde una perspectiva completamente diferente en la historia del problema de la propagación sucesiva de la luz: se piensa que su verdadera contribución para hallar una solución a este interrogante se da indirectamente con el descubrimiento de los satélites de Júpiter. Como se mencionó anteriormente, el astrónomo danés Ole Roemer es considerado como el primero en aportar datos observacionales relevantes en favor de la idea de que la luz requiere de tiempo en su transmisión y dichos datos se derivaron de prácticas de medición que involucraban la ocurrencia de los eclipses de estos satélites.

Con todo lo anterior en mente, el propósito de la presente conferencia es mostrar que el rol de Galileo en la historia de este particular problema de la física-el de la propagación sucesiva de la luz-no se puede reducir únicamente a su contribución indirecta con el descubrimiento de los satélites de Júpiter, y que el experimento por él propuesto no es de ninguna forma inocente. Por el contrario, su aporte es significativo no solo por llevar la búsqueda de su solución de un marco metafísico a uno experimental, sino también por delinear el marco conceptual en el que se inscribirían los trabajos de quienes posterior a su obra se acercaron a este milenario problema. 


\section{Antecedentes históricos}

Se dará comienzo a este recorrido presentando algunos antecedentes históricos acerca del problema de si la luz se transmite de manera instantánea o si requiere de tiempo. En un texto titulado "Medieval Latin Theories of the Speed of Light", el cual se encuentra incluido en la recopilación editada en conmemoración de los 300 años de la presentación de Ole Roemer ante la Academié des Sciences de la propuesta acerca de la propagación sucesiva de la luz, David C. Lindberg -uno de los historiadores de la ciencia más reconocidos del siglo XX- afirma que:

[...] aquellos que estuviesen interesados en el problema [de si la transmisión de la luz es instantánea o requiere de tiempo], ya fuera en 1400 [d.C.] o 400 [d.C.], no tenían más alternativa que buscar una solución que reconciliará con armonía diversas autoridades; derivando la respuesta de las teorías actuales de la naturaleza de la luz o, si esto resultaba ser no concluyente o poco persuasivo, derivándola de las doctrinas establecidas acerca de la naturaleza de Dios y el universo; o tratando de encontrar dicha solución mediante argumentos matemáticos o lógicos que demostraran la necesidad o el absurdo de alguna de las dos posibilidades (Lindberg 67; el énfasis y la traducción son mías).

Lo que expresa el apartado anterior es la manera en que los pensadores de la antigüedad y la medievalidad, hasta más o menos el primer tercio del siglo XVII, se enfrentaban al problema de la propagación sucesiva de la luz, esto es, cuál era la estrategia de los filósofos naturales para dar respuesta al interrogante de si la luz se transmite de manera instantánea o si requiere de tiempo. Lindberg sostiene que el enfoque utilizado apelaba, en primera instancia, a la naturaleza de la luz, esto es, primero establecían teorías acerca de qué era la luz -cuál era su naturaleza- y a partir de ello derivaban si ella se transmite instantáneamente o requiere de tiempo.

Fue común en los periodos históricos mencionados anteriormente que se concluyera que la luz se transmite de manera instantánea, debido principalmente a la influencia del pensamiento aristotélico. Aristóteles pensaba que la luz no era una emanación material de la fuente luminosa sino un estado o cualidad adquirida por el medio. De esta manera, dicha 
adquisición de la cualidad lumínica no ocurría de forma progresiva o sucesiva por parte del medio sino en un solo instante.

A pesar de la influencia del estagirita en la creencia de la transmisión instantánea de la luz, también hubo personajes que llegaron a pensar que la luz requería de tiempo en su transmisión. Uno de los casos más conocidos es el del filósofo árabe Ibn al-Haythem -Alhacén o Alhazen- quien en el siglo XI de nuestra era registraba que la luz no se transmite instantáneamente sino que es un movimiento, y como tal requiere de tiempo.

A comienzos del siglo XVII, Johannes Kepler -uno de los grandes estudiosos de la óptica en los albores de la ciencia moderna-, no solo continuaba considerando que la luz se transmitía instantáneamente, sino que empleaba la misma estrategia argumentativa que se expuso previamente -siguiendo las palabras de Lindberg-. Lo anterior puede verse en la quinta proposición del capítulo primero de su Ad Vitellionem Paralipomena:

El movimiento de la luz no ocurre en el tiempo sino en un momento. Como ha sido demostrado por Aristóteles en los libros sobre el movimiento, existe una cierta proporcionalidad de tiempo a la razón que existe entre el poder motor y el peso del medio. Pero esta fuerza motora posee una razón infinita con respecto a la luz que va a ser movida, debido a que la luz no tiene materia, y por lo tanto, carece de peso. De esta forma, el medio no ofrece resistencia a la luz porque ella carece de materia. En consecuencia, la rapidez de la luz es infinita (Kepler 21; cap. 1, prop. 5; la traducción y el énfasis son míos).

Ejemplos como este se pueden rastrear en la antigüedad griega y la medievalidad, pero interesa aquí resaltar este en particular por su proximidad temporal con el diálogo galileano que se estudiará más adelante -se encuentran separados 34 años-. Con esto en mente, en dicha proposición se puede ver claramente que Kepler argumenta que al ser inmaterial la luz -una consideración acerca de su naturaleza- se sigue que ella debe tener una rapidez infinita, es decir, que debe transmitirse de manera instantánea. Por lo tanto, al considerar primero una característica o propiedad de la naturaleza de la luz -su inmaterialidad-, deriva posteriormente la respuesta concerniente al problema de su transmisión -su transmisión instantánea-. 


\section{Galileo y la naturaleza de la luz}

Lo anterior resulta útil para poner en contexto cuál es el problema con el que se enfrentó Galileo y la estrategia para darle respuesta que él propuso. No obstante, antes de estudiar en profundidad dicha estrategia, será conveniente realizar una salvedad histórica: cuando se habla de Galileo en la historia de la ciencia, por lo general se hace referencia a sus publicaciones y contribuciones en el área de la mecánica, la astronomía observacional -la invención del telescopio y los descubrimientos realizados con este instrumento-, el fortalecimiento del copernicanismo o las controversias con la Iglesia. Poco se habla acerca de Galileo en relación con el estudio de la luz.

Sin embargo, la profesora Susana Gómez ha realizado un copioso trabajo de investigación acerca de las aproximaciones de Galileo al problema de la naturaleza de la luz. Una de sus ideas principales es que las declaraciones o manifestaciones públicas de Galileo en torno a la naturaleza de la luz son significativamente escasas en comparación con otros problemas por los que el pensador italiano se interesó (Gómez, "Galileo" 403). A pesar de lo anterior, en el trabajo de Gómez se evidencia que es posible rastrear el interés de Galileo en su correspondencia privada por la luz y sus consideraciones acerca de ella. Con lo anterior en mente, resulta llamativa la siguiente afirmación que se encuentra en un una carta que Galileo envía a Liceti el 25 de agosto de 1640: "Me habría arriesgado a estar en la cárcel a pan y agua durante toda mi vida con tal de haber tenido la certeza de conseguir un conocimiento tan deseado por mí [como lo era el de la esencia de la luz]" (ctd en Gómez, "Galileo" 418).

A lo largo de la correspondencia de Galileo es posible encontrar algunas claves acerca del pensamiento que este pensador fue tejiendo acerca de la naturaleza de la luz. Uno de sus primeros acercamientos al problema de la luz se da entre los años 1610 y 1613, derivado de uno de sus descubrimientos astronómicos, a saber, las manchas solares. En correspondencia que sostuvo con Benedetto Castelli durante este periodo -argumenta Gómez- se pueden apreciar dos elementos: por un lado, que Galileo consideraba que la luz del sol es una emanación de partículas 
y, por otro, que las manchas solares -observadas por el italiano- son el combustible que permite la regeneración de la materia solar (Gómez, "Galileo" 405-408). Otro acercamiento al problema de la luz se dio con el estudio de la piedra de Bolonia, la cual al ser calcinada y expuesta a la luz del sol, tenía la capacidad de emitir luz en la oscuridad. Esta piedra fue llevada por Galileo a Roma en el año de 1611 y, de acuerdo con la narración de Giulio Cesare Lagalla, su propósito era demostrar la falsedad de la idea aristotélica de luz como una propiedad o cualidad adquirida instantáneamente por el medio y robustecer la idea de ella como una emisión de pequeñas partículas que se desplazaban con una velocidad elevada (Gómez, "Galileo" 408-9).

En este sentido, son claras las conexiones de estas ideas con el pensamiento neoplatónico y atomista que estaba siendo discutido por contemporáneos y predecesores de Galileo. En consecuencia, la profesora Gómez señala que es plausible pensar que Galileo nunca se involucró de manera abierta y pública en discusiones acerca de la naturaleza de la luz debido a que sus creencias e ideas en relación con este problema estaban enmarcadas en el pensamiento neoplatónico y atomista, el cual para aquella época empezaba ser visto cada vez con mayor recelo y con ciertas sospechas de herejía.

Adicionalmente, el pensamiento galileano acerca de la luz tuvo una estrecha relación con otro de sus intereses, a saber, el de la composición de la materia. En este sentido, es importante destacar que las creencias de Galileo en lo concerniente a la composición de la materia se encuentran fuertemente influenciadas por el neoplatonismo y el atomismo. Desde esta perspectiva, resulta interesante que una de las pocas aproximaciones de Galileo al problema de la luz en el ámbito público es el del experimento para intentar dar respuesta al milenario problema de la propagación sucesiva, ya que es precisamente en una discusión acerca del problema de la composición de la materia que aparece la descripción del experimento que se expondrá a continuación. 


\section{Galileo y su experimento para resolver un interrogante milenario}

Galileo se refiere al problema de la propagación sucesiva de la luz en la jornada primera de su libro Consideraciones y demostraciones matemáticas sobre dos nuevas ciencias (en adelante Discorsi). Esta primera jornada transcurre con la discusión acerca de las causas de la resistencia de los cuerpos sólidos a su fractura o rompimiento. En esencia, lo que se trata de responder es por qué si dos cuerpos tienen la misma forma geométrica se comportan de manera diferente debido a su tamaño. Galileo -a través de las palabras de Salviati ${ }^{4}$ - sugiere que la cohesión de los cuerpos sólidos se debe a la existencia de infinitos vacíos indivisibles, lo cual lo lleva a la presentación de algunas consideraciones matemáticas acerca de los infinitos y los indivisibles. Tras esto, se discute si no es posible que los fluidos solo sean la separación de la materia en todas sus partes indivisibles. Al alcanzar este punto se pone en consideración la acción del fuego y de la luz sobre los sólidos. Allí, recordando la historia de Arquímedes y cómo supuestamente utilizaba espejos cóncavos que concentraban los rayos del sol para prender fuego a las embarcaciones de tropas invasoras, Sagredo -otro de los interlocutores del diálogo-se pregunta si la acción de la luz sobre los sólidos puede pensarse sin que intervenga el movimiento o ha de ser el resultado de un movimiento velocísimo.

Se ve aquí que lo que se discute en la primera jornada del diálogo galileano es cómo está compuesta la materia y cuál es la acción de la luz sobre los cuerpos sólidos, además de pensar la conexión entre la luz y el fuego. Por lo tanto, existe una relación entre las consideraciones neoplatónicas que atravesaban el pensamiento de Galileo sobre la estructura de la luz y el

4 Es valioso recordar que los tres interlocutores que participan en los diálogos galileanos tienen un significado en el que se representa una postura de la filosofía natural de la época: Salviati representa al mismo Galileo, y a través de este personaje es que el pensador italiano expresa sus propias ideas; Sagredo personifica al espíritu culto y educado de su época que, no obstante, no se ha adentrado en el estudio de las matemáticas ni se encuentra familiarizado con las últimas ideas y descubrimientos; y Simplicio encarna al filósofo peripatéticoescolástico que constantemente invoca las opiniones y argumentos aristotélicos. 
contexto en el que aparece el experimento para resolver el problema de la propagación sucesiva de la luz. Lo anterior lleva a la profesora Susana Gómez a afirmar lo siguiente:

Estas consideraciones también prueban que para Galileo discutir acerca de la velocidad de la luz no significaba que estuviese omitiendo el problema de su composición atómica, dado que ambas cuestiones se encontraban relacionadas. Lo anterior se hace claro si atendemos al tipo de problemas que los tres protagonistas de los Discorsi discutían antes y después de las breves páginas que se dedican a la luz, a saber, la composición de los fluidos, y los fenómenos de condensación y rarefacción (Gómez, "From a Metaphysical" 214; la traducción es mía).

En consecuencia, hay una conexión entre las consideraciones galileanas en torno a la composición de la materia y sus preocupaciones acerca de la naturaleza de la luz, y este es precisamente el contexto en el que aparece el experimento que aquí se está estudiando.

Frente al problema enunciado por Sagredo en el diálogo galileano -esto es, si la acción de la luz sobre los sólidos puede pensarse sin que intervenga el movimiento o ha de ser el resultado de un movimiento velocísimo-, Salviati señala que siguiendo lo que ha visto en otras acciones sobre los sólidos, esta no puede pensarse como una acción sin movimiento. Precisamente, en esta coyuntura Sagredo introduce la cuestión de si tal movimiento debe ser instantáneo o si acaso requiere de tiempo. Respecto a esta cuestión, el tercer interlocutor del diálogo, Simplicio, afirma que el movimiento de la luz debe ser instantáneo debido a que la experiencia común muestra que en la explosión de un cañón su resplandor llega antes que el sonido. No satisfecho con esta respuesta, Sagredo replica que de esa experiencia solo se puede concluir que el movimiento de la luz es mucho más rápido que el del sonido, no que sea instantáneo. Es aquí que Salviati sugiere un experimento que él mismo ha diseñado para dar respuesta a este interrogante y procede a dar una descripción detallada del mismo:

Dos hombres toman una luz cada uno y la esconden en el interior de una linterna o cualquier otro aparato capaz de cubrirla, de modo que puedan ir encendiéndola y apagándola, poniendo la mano delante, en cuanto ven a su compañero. Después, colocándose uno enfrente del otro a la distancia de unos pocos codos, empiezan a encender y a 
apagar la luz según la siguiente regla: cada vez que uno de los dos ve la luz del otro, enciende inmediatamente la suya. Después de algunos ensayos, se habrá ajustado la maniobra hasta el punto que, sin error posible, en cuanto uno encienda le responderá inmediatamente el otro, de forma que en el momento que una encienda su luz, verá al mismo tiempo aparecer ante sus ojos la de su compañero. Asegurada esta práctica en esta distancia tan pequeña, coloquemos ahora a estos hombres, y con dos luces semejantes, a la distancia de dos o tres millas el uno del otro. Comenzando de noche la misma experiencia, van observando atentamente si las respuestas a sus respectivos encender y apagar guardan, a esta distancia, la misma cadencia que antes. Si es así, se podrá concluir, con bastante seguridad, que la expansión de la luz es instantánea, ya que si ésta necesitara tiempo en una distancia de tres millas, si tenemos en cuenta la ida de una y la vuelta de la otra, la demora tendría que ser suficientemente observable. Y si se repitiese la experiencia con distancias mayores, con ocho o diez millas de distancia, por ejemplo, nos podríamos servir del telescopio, colocando a los observadores con cuidado en el lugar en el que habría de practicarse por la noche la experiencia. Las luces, aunque no fuesen muy potentes, lo que las haría invisibles a simple vista dada la distancia, podrían, sin embargo, encenderse y apagarse fácilmente gracias a la ayuda del telescopio, que ajustaría debidamente las apariciones y desapariciones de la luz, posibilitando así la visión. (Galileo 123-124; §88)

En términos generales, el experimento galileano para determinar si la luz se transmite de manera instantánea o requiere de tiempo resulta metodológicamente sencillo. Lo que sugiere Galileo es situar dos hombres, uno frente al otro, separados por una distancia determinada con anterioridad, cada uno con una lámpara cubierta. El primer hombre abre la pantalla que cubre su lámpara y el segundo hace lo mismo en cuanto percibe la luz de la lámpara de su compañero; el primer hombre debe medir, entonces, el tiempo que transcurre entre la apertura de su propia lámpara y la detección de la señal luminosa de su compañero.

Es importante comprender que el intervalo temporal que se miden en el experimento descrito por Salviati no necesariamente corresponde con el tiempo que tarda la luz en realizar el recorrido. En el caso extremo de que la luz se transmitiera instantáneamente, debería medirse un intervalo temporal en la realización de todo el experimento, el cual correspondería al tiempo de reacción del segundo hombre para abrir la pantalla de su 
lámpara tras detectar la señal lumínica de su compañero. En este sentido, la primera parte del experimento -cuando están separados por la distancia de una milla- consiste en calibrar la cadencia entre sendas acciones y medir con precisión dicho tiempo de reacción.

Luego se repite el mismo procedimiento aumentando paulatinamente la distancia que separa a los dos participantes del experimento. Si al comparar los intervalos temporales medidos con diferentes distancias se mantienen iguales, esto significa que la luz se transmite instantáneamente debido a que lo único que se estaría midiendo es el tiempo de respuesta del segundo experimentador; por el contrario, si hay un aumento de los intervalos temporales a medida que se incrementa la distancia que separa los dos hombres, esto implicaría que la luz requiere de tiempo en su transmisión.

Visto de manera retrospectiva, se puede comprender la acusación de inocencia del experimento de Galileo, ya que no solamente tenía que tener en cuenta lo increíblemente rápida que es la transmisión de la luz -como se conoce en la actualidad-, sino también el tiempo de reacción de quienes están involucrados en la experiencia, el cual es realmente corto (dicho intervalo temporal no se logra medir con exactitud sino hasta finales del siglo XIX y comienzos del XX). De esta manera, el problema con el que se enfrentaba Galileo era la medición de intervalos temporales significativamente cortos. El desarrollo de instrumentos que permitieran medir con precisión estos intervalos no se iba a conseguir sino dos siglos más tarde. Hay que tener en mente que los instrumentos con que se contaba para medir duraciones -esto es, intervalos temporales- en aquel entonces eran bastantes precarios: relojes de arena y clepsidras.

\section{El resultado no-concluyente del experimento galileano}

Tras presentar el diseño del experimento, el mismo Salviati señala que solo ha llevado a cabo el experimento en distancias cortas. Por esta razón se abstiene de ofrecer una conclusión definitiva con respecto a la transmisión instantánea de la luz, y tan solo refiere que, de no ser este el caso, su propagación debe ser velocísima. Lo anterior se puede observar con 
claridad en el siguiente extracto del diálogo: "La verdad es que yo sólo he realizado la experiencia a poca distancia; es decir, a menos de una milla, por lo que no he podido asegurarme de si la aparición de la luz es instantánea. Pero si no es instantánea, he constatado que es velocísima, por no decir momentánea" (Galileo 124; §88-89; énfasis añadido). En otras palabras, la respuesta a la que llega Galileo a través del experimento descrito en los Discorsi es no-concluyente, es decir, no logra extraer una respuesta robusta que excluya definitivamente la otra posibilidad; reconociendo igualmente que la experiencia ni siquiera fue realizada en distancias mayores a una milla. Esta última circunstancia permite pensar en las dificultades enfrentadas en la realización de este experimento.

\section{Intermezzo: ¿Se realizó "realmente" el experimento?}

Antes de continuar, se considera pertinente realizar un corto paréntesis en la exposición y reflexionar sobre el siguiente interrogante: ċse realizó realmente el experimento galileano para determinar si la luz se transmite instantáneamente o si requiere de tiempo? Como herencia de la tradición historiográfica de Alexandre Koyré -historiador que dedicó gran parte de sus investigaciones al estudio de la revolución científica y, en especial, de la obra de Galileo-, es común que cuando se piensa en la figura del pensador italiano no se le piense como un experimentador. Por el contrario, como consecuencia del trabajo de este historiador, se ha atrincherado la creencia de que los experimentos que se encuentran en sus obras no son más que experimentos mentales o experiencias que nunca fueron llevadas a cabo.

Si se tiene en cuenta lo anterior, no existe ningún registro que permita establecer con certeza si Galileo realizó efectivamente dicho experimento. Sin embargo, existe una anotación en el Saggi di Naturali Esperienze de la Accademia del Cimento acerca de la realización de tres experiencias similares a la descrita por Galileo. Dicha academia fue una de las primeras comunidades científicas, cuyo trabajo estuvo fuertemente inspirado en las enseñanzas galileana y cuyos integrantes sí llevaron a cabo una serie de experimentos, muchos de los cuales son similares a los descritos por Galileo en sus obras. De esta manera, en la descripción del experimento 
número 265 de los Saggi -que es una lista de los experimentos ejecutados por la Accademia acompañada de una descripción de cómo se realizaron y cuáles fueron los resultados obtenidos- se encuentra el siguiente reporte de los resultados alcanzados tras la realización de las tres experiencias señaladas con anterioridad:

En una distancia de una milla (que realmente son dos, ya que una de las señales luminosas debe realizar el viaje de ida y la otra el de regreso) no hemos sido capaces de detectar ninguna [demora]. Si es posible percibir de manera exitosa algún retraso sensible a una distancia mayor es un experimento que no hemos podido realizar hasta el momento (Middleton 124; §CCLXV; la traducción es mía).

A pesar de la existencia de esta anotación, queda todavía la inquietud de si Galileo realizó -o intentó realizar- el experimento que describió a través de la voz de Salviati, o en caso de que no lo haya llevado a cabo, cómo fue que llegó a la conclusión expresada en los Discorsi. Es probable que nunca sea posible hallar una respuesta a estos interrogantes. No obstante, es significativo encontrar que durante la segunda mitad del siglo XVII la Accademia del Cimento intentó en diferentes ocasiones la realización de dicho experiencia, llegando al mismo resultado no-concluyente señalado por el pensador italiano.

\section{Algunasconsideraciones en torno al experimentogalileano}

Tras haber presentado la descripción del experimento galileano -expresado mediante la voz de Salviati en los Discorsi- y haber profundizado en su concepción metodológica, en lo que sigue se realiza un análisis epistemológico teniendo como base el trabajo del profesor Alejandro Cassini.

En primer lugar, es importante destacar que el propósito del experimento propuesto por Galileo no es medir la velocidad de la luz, sino determinar si su transmisión es instantánea o requiere de tiempo. Si bien ambos problemas se encuentran relacionados, no son exactamente el mismo. De esta manera, es cierto que si en una práctica se midiera la velocidad de la luz, el segundo problema -el de su propagación- sería resuelto. Sin 
embargo, este no es el enfoque del experimento propuesto por el italiano ${ }^{5}$. Como se estableció en la descripción anterior del experimento, este no requiere en ningún momento de la determinación de un valor numérico de la velocidad de la luz, sino de la comparación de distintos intervalos temporales entre dos eventos -la apertura de la pantalla de la lámpara del primer experimentador y la detección de la señal luminosa del segundopara diferentes distancias. Precisamente, de la comparación de los intervalos temporales medidos es que se puede extraer una conclusión concerniente a las dos posibilidades acerca de la propagación de la luz: si son iguales, la luz se transmite instantáneamente; si divergen, la luz requiere de tiempo en su transmisión. De lo anterior, se desprende, además, que este experimento galileano puede ser considerado como uno de los primeros experimentos cruciales de la historia de la ciencia. En palabras de Cassini: "[l]a finalidad de esta clase de experimentos era contrastar simultáneamente dos hipótesis o teorías rivales, de tal modo que, si el experimento resultaba exitoso, una de las dos debía ser refutada" (119-120).

En segunda instancia, un "resultado negativo" del experimento galileano -esto es, que no se detecte un incremento de los intervalos temporales a medida que se aumenta la distancia entre los experimentadores- no probaría la transmisión instantánea de la luz. Lo anterior se debe a que, frente a dicho resultado, debe considerarse la posibilidad de que la luz se transmite lo suficientemente rápido como para que los intervalos temporales a medir sean significativamente cortos, de tal forma que los instrumentos involucrados en dicha medición no tengan el suficiente grado de apreciación para detectarlos. Si se considera esto con detenimiento, el experimento solo resulta ser crucial para probar que la luz requiere de tiempo en su transmisión -y refutar la transmisión instantánea- al detectarse un cambio en los intervalos temporales; en el caso contrario no se tendría certeza, puesto que debe contemplar una hipótesis auxiliar, a saber, que haya una causa técnica por la que no se pudo detectar el cambio

5 Cabe resaltar que a lo largo del siglo XVII y comienzos del XVIII el problema al que trataban de dar solución los filósofos naturales era el de si la luz se transmite instantáneamente o si requiere de tiempo, en este sentido, su objetivo nunca fue medir su velocidad. A pesar de esto, una tradición historiográfica -particularmente de los libros de texto de física y ópticacontribuyó a que se atrincherara la creencia de que Galileo fue el primero en intentar medir la velocidad de la luz, y que Roemer y Bradley fueron los primeros en conseguirlo. 
en los intervalos temporales. En definitiva, con el experimento descrito en los Discorsi no se podría refutar la propagación sucesiva de la luz.

Finalmente, y en relación con el primer elemento destacado en este breve análisis epistemológico, el experimento galileano no requiere del concepto de velocidad de la luz ni de su medición directa, a lo sumo, se puede argumentar que este posibilita una medición indirecta. En ese sentido, resulta valiosa la siguiente afirmación del profesor Cassini:

[El experimento de Galileo] [n]o requiere, además, la noción de velocidad de la luz, sino solo determinar si su propagación requiere de tiempo. El resultado del experimento se puede describir exclusivamente en términos de los conceptos de distancia y duración. [...] De manera más general, el resultado de cualquier experimento del tipo de Galileo, no importa cuán grande sea la carga teórica instrumental que tenga, es decir, cuán sofisticado sean los instrumentos de medición empleados, puede describirse sin emplear más que conceptos cinemáticos (141; el énfasis es mío).

En consecuencia, para poder realizar el experimento de Galileo -como ha sido descrito con anterioridad- lo único que se requiere medir es la distancia que separa a los dos participantes y el intervalo temporal entre la apertura de la lámpara del primer experimentador y el momento en que se detecta la señal luminosa de la lámpara del segundo. De este modo, en la realización de este experimento se necesita únicamente de las nociones de distancia y de tiempo, no del concepto de velocidad. En términos un tanto más coloquiales, para llevar a cabo el experimento de los Discorsi tan solo se requiere contar con un metro lo suficientemente largo para medir la distancia entre los experimentadores y un reloj con el suficiente grado de apreciación para medir los intervalos temporales involucrados. Es cierto que al obtener estas mediciones se podría dar un paso más y calcular la velocidad de la luz, pero no es necesario. En suma, para el experimento es suficiente con medir distancias y duraciones.

\section{Galileo y la mecanización de la luz}

Lo anterior resulta importante si se tiene en mente la siguiente reflexión de la profesora Susana Gómez: "¿No deberíamos, quizás, hablar [en el 
pensamiento galileano] de un intento de mecanizar la luz, la cual hasta ese momento había sido sólo tratada metafísicamente por los filósofos neoplatónicos [y aristotélicos] o matemáticamente mediante la óptica geométrica?" (Gómez, "From Metaphysical" 202; la traducción y el énfasis son míos). Una de las ideas sugeridas por esta investigadora en sus escritos sobre Galileo es que en las reflexiones de este pensador sobre la naturaleza de la luz hay un intento de mecanización de este fenómeno, esto es, que el pensador italiano -siguiendo las ideas neoplatónicasconcebía la luz como una emanación de diminutas partículas, las cuales eran susceptibles de ser estudiadas mediante la mecánica como cualquier otro cuerpo. En este sentido, argumenta la profesora Gómez que:

El caso de la naturaleza de la luz es un buen ejemplo del hilo de continuidad (no de identidad) que conecta las diferentes fases del atomismo galileano: desde las afirmaciones sobre las manchas solares hasta las páginas de los Discorsi acerca de la velocidad de la luz, no encontramos una renuncia acerca de las ideas fundamentales o los conceptos [sobre la naturaleza de la luz], ni una transformación del propósito de comprender su naturaleza mediante un estudio experimental y cuantitativo de su velocidad ("Mechanization" 211; la traducción y el énfasis son míos).

Con todo lo que se ha expuesto en esta conferencia se puede asegurar que el interés galileano por el problema de la propagación sucesiva de la luz se inserta en el marco de la mecanización de la luz sugerida por Susana Gómez desde las dos ideas resaltadas mediante el uso de cursivas en la cita anterior. Por un lado, con una transformación del enfoque tradicional para abordarlo, esto es, no partiendo de teorías metafísicas acerca de la naturaleza de la luz sino teniendo como base un estudio experimental que posibilite luego conocer la naturaleza de la luz. Por otro lado, con un tratamiento cuantitativo -tanto métrico como matemáticode las nociones de distancia y duración.

\section{A manera de conclusión: sobre hombros de gigantes}

Para dar cierre a esta conferencia, se quiere realizar una reflexión final considerando el experimento galileano para dar respuesta al problema de 
si la luz se transmite instantáneamente o si requiere de tiempo en relación con el nombre de este evento, a saber, Sobre hombros de gigantes.

Se había comentado con anterioridad que, en la historia de la ciencia, quien se considera ser el primero en dar una respuesta de carácter observacional a este problema es el astrónomo danés Ole Roemer en el año de 1676. De esta manera, en Septiembre de dicho año Roemer anunció a los miembros de la Académie des Sciences que el próximo eclipse del primer satélite de Júpiter que se esperaba para el 9 de Noviembre ocurriría 10 minutos más tarde de lo que se esperaba de acuerdo con las tablas de los movimientos de los satélites jovianos elaboradas por Gian Domenico Cassini. Dicha predicción se comprobó el día del eclipse en el Observatoire Royal de París, y el día 21 del mismo mes el astrónomo danés tuvo la oportunidad de exponer ante los miembros de la academia un trabajo en el cual sugería que dicho retraso se debía a la necesidad de introducir una nueva ecuación -ecuación de la luz- en la elaboración de las tablas de los movimientos de los satélites de Júpiter y en el cálculo de la ocurrencia de sus eclipses. Dicha ecuación, argumentaba Roemer, se derivaba del hecho de que la luz requería de tiempo para su transmisión desde el satélite hasta la Tierra y que, de acuerdo con sus cálculos, la luz tarda alrededor de 22 minutos en atravesar el diámetro de la órbita terrestre.

Más allá de esta sucinta descripción de los hallazgos de Roemer, resulta interesante detener la mirada sobre las palabras con las que se abre la comunicación pública de esta intervención en el Journal des Sçavans:

Los filósofos [naturales] han estado trabajando durante muchos años en decidir por medio de alguna experiencia si la acción de la luz es transmitida en un instante a lugares remotos o si, por el contrario, requiere de tiempo. Mr. Rømer de la Real Academia de las Ciencias ha ideado una manera, a partir de las observaciones del primer satélite de Júpiter, por la cual demuestra que para una distancia de unas 3000 ligas [...] la luz requiere menos de un segundo [en su transmisión] (Roemer 377; la traducción y el énfasis son míos).

El recorrido que se ha hecho en esta conferencia ha mostrado que por casi dos milenios la estrategia tradicional de enfrentarse a este problema 
por parte de los filósofos naturales fue establecer primero teorías acerca de la naturaleza de la luz y de ellas derivar una posible respuesta. Sin embargo, el apartado resaltado con cursiva en la anterior cita permite apreciar cuál fue el enfoque utilizado por los filósofos naturales en los años que preceden al trabajo del astrónomo danés para dar respuesta al problema de la propagación sucesiva de la luz, a saber, "[...] decidir por medio de alguna experiencia" (Roemer 377).

72 años separan la publicación de los hallazgos de Roemer del Paralipomena de Kepler, y 38 años la separan de la aparición de los Discorsi de Galileo. Kepler es un ejemplo paradigmático del enfoque tradicional quizás uno de los últimos filósofos naturales en enfrentarse al problema de esta manera- al problema de la transmisión instantánea y Roemer es considerado el primero en dar una respuesta de carácter observacional en favor de la propagación sucesiva de la luz. Lo anterior permite apreciar que durante el periodo que separan a estos dos pensadores se efectuó un cambio profundo en la estrategia para intentar resolver el interrogante sobre el que se ha centrado la atención en esta charla. De igual forma, es posible establecer que en ese periodo de 72 años el trabajo de Galileo Galilei contribuyó significativamente a dicha transformación: el italiano no sólo descubre los cuatro satélites de Júpiter cuyo estudio detallado llevó a Roemer a sus hallazgos, sino que las ideas metodológicas expresadas en la primera jornada de los Discorsi -en voz de Salviati- son similares a las empleadas por el astrónomo danés: utilizar telescopios, medir intervalos temporales, y considerar cambios en las distancias entre una fuente luminosa y un observador. Igualmente, a lo anterior hay que añadir que se prescinde del concepto de velocidad de la luz.

Precisamente esto es lo que se ha deseado resaltar en esta conferencia, a saber, que la contribución de Galileo Galilei a la solución del problema de la propagación sucesiva de la luz no es únicamente indirecta -mediante el descubrimiento de los satélites de Júpiter-, sino que el experimento que propuso el filósofo natural italiano en los Discorsi estableció un nuevo marco de referencia para la búsqueda de una solución para dicho problema, esto es, un marco de carácter experimental que tuvo como base los conceptos métricos de distancia y duración. 


\section{Lista de referencias}

Cassini, Alejandro. "El experimento crucial de Galileo: un análisis epistemológico". Análisis Filosófico 34.2 (2014): 119-45.

Cohen, I. Bernard. "Roemer and the First Determination of the Velocity of Light (1676)". Isis 31.2 (1940): 327-79. Jstor. Web.

Galilei, Galileo. Consideraciones y demostraciones matemáticas sobre dos nuevas ciencias. Eds. Carlos Solís y Javier Sádaba. Madrid: Editora Nacional, 1981. Impreso. (Obra original publicada en 1638 bajo el título Discorsi e dimostrazioni matematiche, intorno à due nuove scienze)

Gómez, Susana. "From a Metaphysical to a Scientific Object: Mechanizing Light in Galilean Science”. Eds. Daniel Garber y Sophie Roux. The Mechanization of Natural Philosophy. s. I.: Springer, 2013. 191215. Web.

- "Galileo y la naturaleza de la luz". Eds. José Montesinos y Carlos Solís. Il largo campo del filosofare: Eurosymposium Galileo 2001. La Orotava: Fundación Canaria Orotava de Historia de la Ciencia, 2001. 403-18.

. "The Mechanization of Light in Galilean Science". Galileana: Journal of Galilean Studies 5 (2008): 207-44. Web. <http://bibdig. museogalileo.it/Teca/Viewer?an=917416>

Kepler, Johannes. Optics. Paralipomena to Witelo E Optical Part of Astronomy. Trad. William H. Donahue. Santa Fe: Green Lion Press, 2000. (Obra original publicada en 1604 bajo el título Ad Vitellionem paralipomena. Astronomiae pars optica)

Lindberg, David. C. "Medieval Latin Theories of the Speed of Light". Ed. René Taton. Roemer et la vitesse de la lumière. Paris: Libraire Philosophique J. Vrin, 1978. 45-72. Impreso.

Middleton, W. E. Knowles. The Experimenters: A Study of the Accademia del Cimento. Baltimore $\mathcal{E}$ London: The Johns Hopkins Press, 1971. Impreso.

Roemer, Ole. "A Demonstration concerning the Motion of Light, communicated from Paris in the Journal des Scavans, and here made in English". Cohen 377-78. (Obra original publicada en 1676, Journal des Sçavans) 